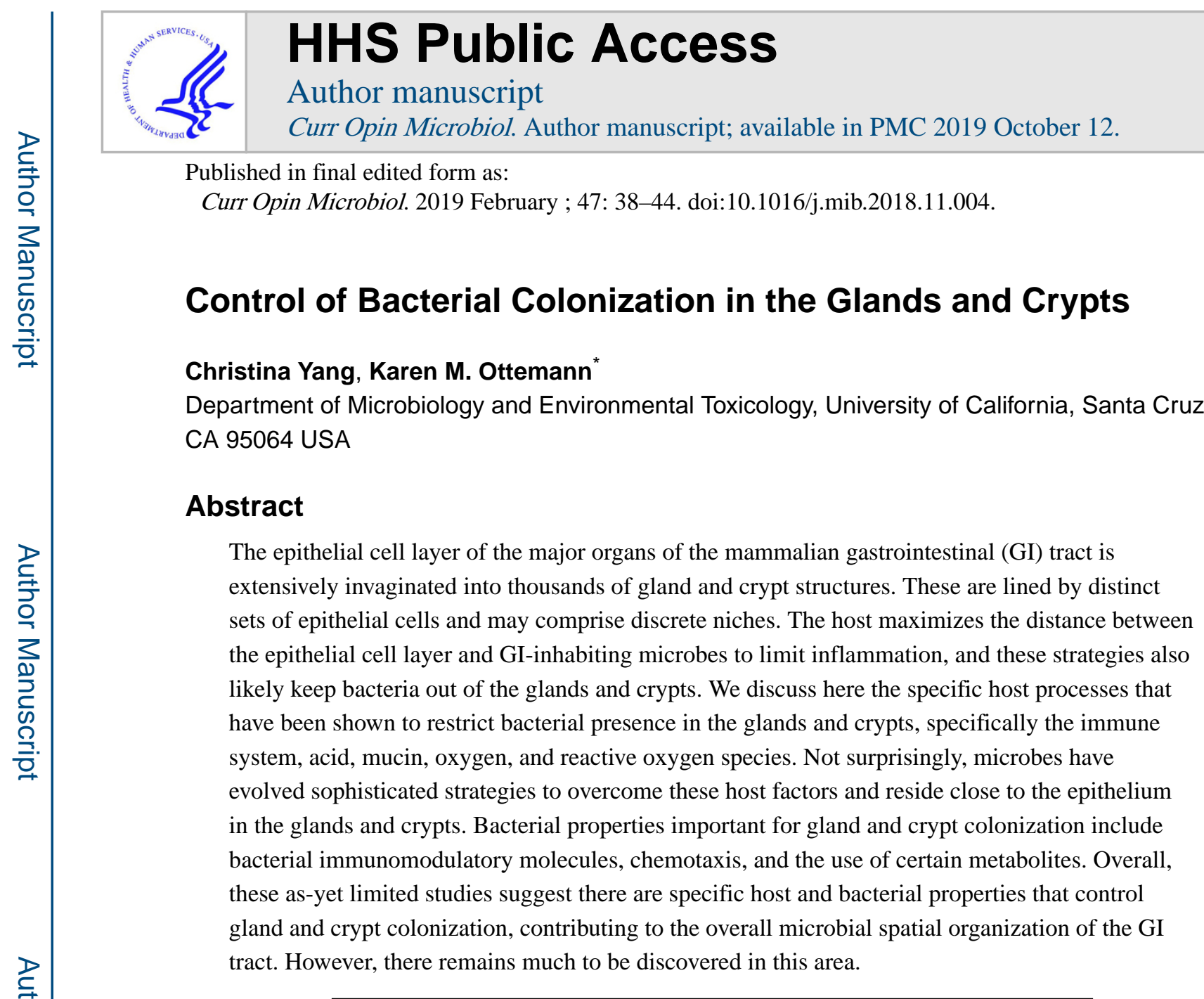

\title{
Introduction
}

The gastrointestinal (GI) tract is a vast organ system with many distinct areas, each harboring a diverse array of microbial species. Many of these microbes reside within the host for long periods of time, indicating strong adaptation. We have accumulated a reasonable understanding of the types of microbes that inhabit the GI tract, but are still learning about their spatial relationships. This information was obtained largely from fecal samples, which lose the original microbial spatial organization [1-5]. The microbial composition varies along the GI tract [6,7] and there are distinct microbial populations that are luminal- and mucosal-associated [8]. A key attribute of the GI tract is the extensive invagination of the epithelial layer to form glandular or crypt structures. Laser capture microdissection has identified specific crypt-associated microbes [9], suggesting there may be multiple niches. However, the factors that influence whether bacteria reside in the different microenvironments are not understood. Because evidence suggests that the glandular structures of the GI tract may provide unique and long term colonization sites, this review focuses on our current understanding of host and bacterial factors that determine localization within these glandular structures.

\footnotetext{
*To whom correspondence should be addressed at Ottemann@ucsc.edu.
} 


\section{Anatomy and Physiology of the GI Tract}

The GI tract is made up of the mouth, esophagus, stomach, small intestine, cecum, appendix, and colon. The epithelial cell layer invaginates into glandular structures that are referred to as glands in the stomach, and crypts in the small intestine, cecum, and colon [10-12]. Each gland or crypt contains stem cells responsible for differentiation of the various epithelial cell types that line the structures (Fig. 1) [10-14].

Within the stomach, there are two types of glands: zymogenic glands of the corpus, and mucous glands in the antrum (Fig. 1) [15]. These glands contain common and unique cell types. Both contain mucous cells that secrete protective mucus, and endocrine cells that secrete hormones for signaling and regulating physiological processes such as digestion and motility (Fig. 1) [10,16]. The zymogenic glands uniquely contain the acid-secreting parietal cells and the pepsinogen-secreting chief cells (Fig. 1) [13,16,17]. Acid is a key difference between zymogenic and mucous glands as discussed below.

The duodenum, jejunum, and ileum make up the small intestine, and all these regions contain crypts of the same type (Fig. 1). The small intestine's main role in the digestive system is to absorb nutrients, and its absorptive surface area is increased through finger-like projections called villi [11]. At the base of a villus, the epithelial cell layer invaginates to form crypts (Fig. 1). These crypts, like glands in the stomach, contain hormone-secreting endocrine cells and mucus-secreting cells, here called goblet cells. They uniquely contain nutrient absorbing enterocytes and Paneth cells [14]. Paneth cells secrete antimicrobial molecules that diffuse to the small intestine lumen [18]. The rest of the intestine, including the large intestine and the cecum, all contain crypts but lack villi (Fig. 1). The cecal and colonic crypts have a similar cellular composition as the small intestine crypts, but lack Paneth cells (Fig. 1) [12,14].

Throughout the GI tract, microbes are kept separated from the underlying lamina propria, the hub of immune activity, to avoid stimulating inflammation. This separation is achieved through the "mucosal firewall" [19], the collective action of mucus, epithelial cells, and the lamina propria immune cells [20]. Generally, the glands and crypts have low levels of microbes as part of this mucosal firewall strategy. Some microbes, however, have evolved the ability to bypass these defenses and stably colonize the glands and crypts [21-25]; how they accomplish this feat is discussed below.

\section{Host factors that affect gland and crypt bacterial colonization}

As described above, mammals have specific mechanisms that keep microbes at a distance from the GI tract cellular surfaces, and likely help to keep the crypts and glands microbefree. There appears to be specific microbiota in the colonic crypts, but the small intestinal crypts are not normally colonized [26]. In the stomach, the pathobiont Helicobacter pylori is the only known gland inhabitant. The next section describes the handful of host factors that have been identified to modulate gland and crypt colonization. 
Acid

Stomach acid is a potent antimicrobial that is produced in the corpus glands, and may limit colonization at that site. Acid is secreted in the form of hydrochloric acid $(\mathrm{HCl})$ by the parietal cells of the zymogenic corpus glands (Fig. 1) [10]. H. pylori colonizes both the corpus and antral glands, but prefers the antral glands during early mouse infection [21]. $H$. pylori senses acid as a chemotaxis repellent [27-29]. This observation has led to the hypothesis that $H$. pylori prefers the antral glands, because it is actively repelled from the acid-producing corpus glands [27-29]. In favor of this idea, omeprazole treatment, which inhibits acid secretion, significantly elevated antral and corpus gland bacterial populations compared to non-treated mice [29]. During the chronic stages of infection, $H$. pylori favors the corpus glands [21], possibly due to its ability to cause gastric atrophy and parallel decreased acid secretion [30]. Overall, these findings suggest stomach acid is an antimicrobial that may regulate gland colonization.

\section{Mucin}

To access the glands and crypts, microbes must bypass the sieve-like mucus layer, which is generated by mucus-secreting cells in the glands and crypts [31-33]. Mucus has been shown to influence intestinal crypt colonization by Vibrio cholerae, a diarrheal-causing pathogen. $V$. cholerae has been seen to inhabit the small intestine crypts via microscopy, with high numbers in the intestinal region closest to the stomach [22]. When mice were treated with $\mathrm{N}$-acetyl-L-cysteine (NAC), a chemical that breaks down mucus, $V$. cholerae crypt colonization increased compared to non-treated mice. These data thus suggest that some property (possibly mucus), is affected by NAC treatment, that restricts crypt colonization. Mucus may play similar roles for other microbes, although this remains to be tested.

\section{Oxygen}

The presence of oxygen close to the epithelial surface also contributes to gland and crypt colonization. The discovery of the importance of oxygen came from studies to define the microbial population of the murine colonic crypts. Pédron et al. used silver nitrate staining to non-specifically stain bacteria and determine that bacteria normally reside in the colonic crypts but not the small intestine ones [26], suggest that the absence of Paneth cells allow bacteria to reside in the colonic crypts. To identify the crypt bacterial species, laser capture microdissection was used to isolate the crypt luminal space coupled by 16s rRNA amplification and sequencing [26]. This analysis showed that the crypt population belonged to the genus Acinetobacter. Acinetobacter are strict aerobes, suggesting that there is oxygen in the crypts $[9,26]$. This oxygen may select for bacterial subpopulations that are able to tolerate and/or require oxygen, in contrast to the many bacteria in the gut that are strict anaerobes.

Other bacteria that utilize oxygen have also been associated with glandular structures. The microaerophilic bacteria H. pylori and Campylobacter jejuni reside in the gastric glands and small intestine crypts, respectively [24,34]. Overall, these results suggest that oxygen is a factor that may contribute to colonization of the glands and crypts throughout the GI tract. 


\section{Immune System Control}

The immune system is another host factor that plays a role in modulating bacterial crypt colonization. The crypt Paneth cells highly express a key immune recognition receptor, Nod2, which is a cytoplasmic receptor that recognizes conserved bacterial peptidoglycan cell wall moieties $[11,35]$. To examine the role of Nod2 in intestinal function, small intestinal crypts were isolated from WT and $\mathrm{Nod}_{2}{ }^{-/}$mice and stimulated with muramyldipeptide (MDP), a Nod2 ligand. After stimulation, the crypt secretions from WT mice killed bacteria, while crypt secretions from Nod2 ${ }^{-/-}$mice were not able to induce the same killing phenotype. This finding suggested that Nod2 regulates the production of secreted antimicrobial factors. Consistent with this idea, Nod2 ${ }^{-1-}$ mice had high colonization of Bacteroides, Firmicutes, and Bacillus commensal genera, and had difficulty clearing Helicobacter hepaticus, an opportunistic murine pathogen that resides in the intestinal crypts [36,37]. These sets of data suggest the presence of microbes induces Nod2-dependent signaling within the crypts, which in turn produces antimicrobial molecules. One idea is that microbes that are able to colonize the small intestinal crypts either poorly activate Nod2 or are more resistant to the resultant secretions.

\section{Reactive Oxygen Species}

Reactive oxygen species (ROS) also seem to play a role in regulating the gland bacterial populations. ROS are generally made by the host defense mechanisms in response to microbial perturbation. This response occurs with $H$. pylori, in which the host upregulates ROS production after infection [38]. ROS are sensed as by the bacterial environmental sensing chemotaxis system as molecules the bacteria swim away from. ROS are sensed by the H. pylori chemoreceptor TlpD, via an unknown mechanism. TlpD mutants are unable to swim away from ROS as $H$. pylori normally would [39,40]. $\Delta$ tlpD mutants colonize fewer glands in the corpus and antrum compared to WT H. pylori, suggesting they poorly access new glands [40]. This defect was rescued when the host was unable to produce ROS including hydrogen peroxide from the epithelial cells and superoxide from the immune cells [40]. This work suggests that reactive oxygen species are a major factor that prevents access to new glands and $H$. pylori overcomes this barrier by using ROS-sensitive chemotaxis.

\section{Bacterial factors shown to contribute to localization of the glands and crypts}

As described above, hosts appear to have mechanisms to limit microbial colonization of the crypts and glands. Microbes, not surprisingly, appear to have developed ways to colonize these niches. Bacterial abilities identified to help microbes live in the glands and crypts are discussed below.

\section{Metabolism}

Bacterial metabolism influences gland colonization. A role for metabolism has been determined in Bacteroides fragilis, a commensal member of the GI microbiota that colonizes the colonic crypts. Germ-free mice mono-colonized with WT B. fragilis could be supercolonized by a different strain but not the same one, a phenomenon termed colonization 
resistance [23]. These results support a model in which $B$. fragilis fully colonizes a saturable niche and prevents colonization by a second strain that colonizes the same niche.

Colonization resistance relied upon a gene cluster that the authors named commensal colonization factor (ccf); this system is homologous to the Sus sugar uptake system [23]. $B$. fragilis mutants lacking the $c c f$ system did not confer colonization resistance and did not colonize the colonic crypts [23]. These results are consistent with the idea that crypts contain a specific nutritional microenvironment that requires particular microbial metabolism. Specific sugar uptake seems to be one of such example. Whether there are other metabolic functions specifically needed in the glands or crypts remains to be determined.

\section{Polysaccharide A}

Another mechanism that supports bacterial crypt localization is by dampening the immune response. This idea has been supported with the colonic crypt colonizer $B$. fragilis, which has a capsular polysaccharide A (PSA) that has immunomodulatory effects [41]. B. fragilis PSA mutants cannot colonize the mouse colonic crypts (based on microscopy), in contrast to WT B. fragilis that robustly colonizes this niche [42]. PSA mutants had high numbers of pro-inflammatory $\mathrm{T}_{\mathrm{H}} 17$ cells, leading to the hypothesis that an inflammatory environment developed that eliminated the $B$. fragilis from the crypts [42]. These results suggest that PSA normally suppresses the $T_{H} 17$ cell population. PSA activates $T_{\text {regs }}$ to secrete IL-10, an antiinflammatory cytokine, via a TLR2-signaling pathway [42]. Therefore a possible mechanism for PSA immune suppression is via activation of $T_{\text {reg }}$ cells that suppress $T_{H} 17$ cells and inflammation, and allow bacteria to reside close to host tissues and within the intestinal crypts.

\section{Motility and Chemotaxis}

Numerous lines of evidence show that bacterial motility is an important factor for gland colonization. Maintaining gland or crypt localization is challenging because of the continuous production of mucus and extrusion of dead cells, and therefore it is thought that crypt localization is an active process, requiring adherence to non-moving cells and/or directed and efficient swimming. Evidence supporting the need for efficient swimming has come from the pathogens $H$. pylori in the stomach, V. cholerae in the small intestine, and $C$. jejuni in the cecum.

C. jejuni is a motile bacterium that localizes to the cecal crypts. Recently, a $C$. jejuni mouse infection model was developed by the knockout of single IgG Interleukin-1 Related Receptor (SIGIRR). SIGIRR ${ }^{-/}$mice develop human-like $C$. jejuni symptoms [43]. $C$. jejuni mutants lacking the $p g p 1$ or $p g p 2$ genes failed to localize like WT to the SIGIRR ${ }^{-/}$mouse crypts [44]. These bacterial mutants are rod shaped, as opposed to the typical spiral shape, due to loss of their ability to modify peptidoglycan. They also exhibit impaired motility in soft agar [44]. These data suggest that a helical shape may be important for motility in the viscous environments of the crypts. In agreement with this idea, many helical-shaped bacteria also reside in GI glandular structures [45-47], including $H$. pylori [34] and $H$. hepaticus [37]. However, it has not been determined whether $H$. pylori and $H$. hepaticus shape is important for gland localization. 
Bacterial motility is controlled by the chemotaxis signaling system, to give directed movement of bacteria in response to chemical gradients [48,49]. These chemical gradients are sensed by chemoreceptors that act via a signal transduction system to influence the rotation of the flagellar apparatus and control directional changes.

H. pylori chemotaxis affects gland colonization. As mentioned above, chemotaxis systems sense environmental signals and use a signal transduction system to control swimming behavior, allowing the bacteria to move toward attractants and away from repellents. Mutants lacking the signal transduction proteins are non-chemotactic $\left(\mathrm{Che}^{-}\right)$, and have significantly less bacteria in the corpus and antrum glands compared to WT during early infection [21,50]. H. pylori has four chemoreceptors that control chemotaxis by sensing a variety of signals [49]. The chemoreceptors TlpB, TlpA, and TlpD sense acid as a key repellent [27-29]. A mutant lacking both TlpA and TlpD $(\triangle t l p A D)$ had significantly fewer bacteria per antral gland compared to WT, suggesting $H$. pylori uses these chemoreceptors to access the glands [29]. As discussed above, H. pylori may use acid to direct it away from the lumen and corpus glands and into the antral ones. Consistent with this idea, chemical inhibition of acid secretion partially rescued the $\triangle t I p A D$ defect [29].

Additional studies have focused on the TlpD chemoreceptor, which senses acid and additionally ROS as repellents [39,40]. $\Delta t l p D$ mutants are defective in spreading across multiple glands in the antrum and corpus [40]. As described above, this defect could be rescued by loss of epithelia that produce $\mathrm{H}_{2} \mathrm{O}_{2}$ via the DUOX enzyme, or the lack of immune cell superoxide via the phagocyte oxidase (Phox) [40]. Thus, this work suggests that ROS are a major factor that prevent gland access. Interestingly, $\Delta c h e Y$ or $\Delta t l p D$ mutants both colonized fewer glands compared to WT H. pylori, but within those glands, they reached approximately WT levels per gland. This phenotype suggests that their defect was mostly in access to the glands, but not in multiplication within glands $[21,40]$. TlpD and TlpA both sense other signals [29,39,51], so further work is needed to dissect the precise signals that modulate gland colonization. Collectively, however, these reports imply that metabolite gradients exist that guide bacteria to colonize the glands.

Conversely, $V$. cholerae uses chemotaxis to avoid the small intestinal crypts. Che ${ }^{-}$mutants displayed a hypercolonization phenotype overall [22,52], with a higher population in the crypts compared to WT [22]. It's not clear whether there are simply more bacteria in the crypts because the overall population is higher, or because $\mathrm{Che}^{-}$mutants lose an ability to avoid crypts. $V$. cholerae may want to avoid the crypts because the small intestine crypts have an inhospitable environment due to antimicrobial-secreting Paneth cells [18,52]. Alternately, V. cholerae may maintain a high luminal population to promote transmission. This difference in how chemotaxis is used may be due to a number of factors, including anatomical differences in the stomach versus the small intestine, as well as the fact that $H$. pylori is a chronically infecting pathogen, in contrast to V. cholerae, which causes acute infection. 


\section{Conclusions}

Several studies have begun to highlight the variety of factors both on the bacterial side as well as the host side that influence gland and crypt colonization. Although these factors individually have been implicated, a mixture of these factors almost certainly combines to dictate whether a microbe can colonize the glands or crypts. Host defenses that protect the glands/crypts include antimicrobials such as acid, antimicrobial peptides and ROS, along with mucus and oxygen. These properties act together to maximize the distance between the microbiota and the epithelium of the glands and crypts [10-12,14]. This distance may be key to protecting the stem cells within the glands/crypts, as these are essential to regenerate the entire epithelial cell population. Unsurprisingly, microbes have evolved to deal with these host mechanisms to stably colonize and persist within the glands and crypts using a combination of chemotactic motility, metabolic abilities, and immunosuppressive skills. Studies have shown bacteria are within the glands during chronic infection [21,23]. Future work will no doubt identify more factors that contribute to gland and crypt colonization. Ultimately, this knowledge will help scientists to engineer bacteria to be able to occupy this niche to develop beneficial microbial therapeutics that are able to establish long term colonization.

\section{Acknowledgements}

We would like to thank Kevin S. Johnson of the Ottemann Lab for his thoughtful comments on this review. The described project was supported by National Institutes of Health National Institute of Allergy and Infectious Disease (NIAID) grant RO1AI116946 and (to K.M.O.). The funders had no role in study design, data collection and interpretation, or the decision to submit the work for publication.

\section{References}

1. Finegold SM, Attebery HR, Sutter VL: Effect of diet on human fecal flora: comparison of Japanese and American diets. Am J Clin Nutr 1974, 27:1456-1469. [PubMed: 4432829]

2. Moore WE, Holdeman LV: Special problems associated with the isolation and identification of intestinal bacteria in fecal flora studies. Am J Clin Nutr 1974, 27:1450-1455. [PubMed: 4215311]

3. Benno Y, Endo K, Mizutani T, Namba Y, Komori T, Mitsuoka T: Comparison of fecal microflora of elderly persons in rural and urban areas of Japan. Appl Environ Microbiol 1989, 55:1100-1105. [PubMed: 2547333]

4. Suau A, Bonnet R, Sutren M, Godon JJ, Gibson GR, Collins MD, Dore J: Direct analysis of genes encoding 16S rRNA from complex communities reveals many novel molecular species within the human gut. Appl Env Microbiol 1999, 65:4799-4807. [PubMed: 10543789]

5. Zoetendal EG, Rajilic-Stojanovic M, De Vos WM, Rajili-Stojanovi M: High-throughput diversity and functionality analysis of the gastrointestinal tract microbiota. Gut 2008, 57:1605-1615. [PubMed: 18941009]

6. Eckburg PB, Bik EM, Bernstein CN, Purdom E, Sargent M, Gill SR, Nelson KE, Relman DA: Diversity of the Human Intestinal Microbial Flora Paul. Science (80- ) 2006, 308:1635-1638.

7. Gill SR, Pop M, DeBoy RT, Eckburg PB, Turnbaugh PJ, Buck SS, Jeffrey GI, Relman DA, FraserLiggett CM, Nelson KE: Metagenomic analysis of the human distal gut microbiome. Sci 2006, 312:1355-1359.

8. Nava GM, Stappenbeck TS: Diversity of the autochthonous colonic microbiota. Gut Microbes 2011, 2:627-38.

9. Pédron T, Mulet C, Dauga C, Frangeul L, Chervaux C, Grompone G, Sansonettia PJ: A cryptspecific core microbiota resides in the mouse colon. MBio 2012, 3:e00116-12. [PubMed: 22617141] 
10. Goldberg E, Raufman J-P: Stomach and Duodenum: Anatomy and Structural Anomalies Yamada's Textb Gastroenterol Sixth Ed 2016, 1:60-72.

11. Rubin DC, Shaker A: Small Intestine: Anatomy and Structural Anomalies Yamada's Textb Gastroenterol Fifth Ed 2016, 1:73-92.

12. Umanskiy K, Matthews JB: Colon: Anatomy and Structural Anomalies Yamada's Textb Gastroenterol Sixth Ed 2016, 1:93-107.

13. Mills JC, Shivdasani RA: Gastric Epithelial Stem Cells. Gastroenterology 2011, 140:412-424. [PubMed: 21144849]

14. van Es JH, Bartfeld S, Clevers H: Stem Cells and Tissue Renewal Yamada's Textb Gastroenterol Sixth Ed 2016,

15. Lee ER, Trasler J, Dwivedi S, Leblond CP: Division of the mouse gastric mucosa into zymogenic and mucous regions on the basis of gland features. Am J Anat 1982, 164:187-207. [PubMed: 7124652]

16. Willet SG, Mills JC: Stomach Organ and Cell Lineage Differentiation: From Embryogenesis to Adult Homeostasis. C Cell Mol Gastroenterol Hepatol 2016, 2:546-559.

17. Perey BJF: Recent Advances in the Physiology of Gastric Acid Secretion. Canad Med Ass J 1963, 89:1183-1188. [PubMed: 14088824]

18. Clevers HC, Bevins CL: Paneth Cells: Maestros of the Small Intestinal Crypts. Annu Rev Physiol 2013, 75:289-311. [PubMed: 23398152]

19. Belkaid Y, Hand TW: Role of the microbiota in immunity and inflammation. Cell 2014, 157:121141. [PubMed: 24679531]

20. MacPherson AJ, Slack E, Geuking MB, McCoy KD: The mucosal firewalls against commensal intestinal microbes. Semin Immunopathol 2009, 31:145-149. [PubMed: 19707762]

21. Keilberg D, Zavros Y, Shepherd B, Salama NR, Ottemann KM: Spatial and Temporal Shifts in Bacterial Biogeography and Gland Occupation during the Development of a Chronic Infection. MBio 2016, 7:e01705-16. [PubMed: 27729513] A study showing the population dynamics of WT and non-chemotactic $H$. pylori within the gastric glands of the stomach antrum and corpus over a 6-month period. WT prefers the antrum glands in the beginning of infection but dominates in the corpus later in the infection. This study provided further support for the finding that chemotaxis is important for gastric gland colonization.

22. Millet YA, Alvarez D, Ringgaard S, von Andrian UH, Davis BM, Waldor MK: Insights into Vibrio cholerae Intestinal Colonization from Monitoring Fluorescently Labeled Bacteria. PLoS Pathog 2014, 10:e1004405. [PubMed: 25275396] This work identified that when mucus is decreased by $\mathrm{N}$-acetyl-L-cysteine (NAC) treatment, $V$. cholerae better colonizes the crypts compared to no treatment. Additionally, Millet et al. observed that deletion of chemotaxis cluster 2 resulted in hypercolonization of the crypts along the small intestine.

23. Lee SM, Donaldson GP, Mikulski Z, Boyajian S, Ley K, Mazmanian SK: Bacterial colonization factors control specificity and stability of the gut microbiota. Nature 2013, 501.This study identified that the commensal colonization factors ( $c c f)$ gene cluster is required for $B$. fragilis to colonize the colonic crypts. Additionally, $c c f$ mutants were unable to replinish the population of bacteria in the mucous layer upon perterbation with antibiotic treatment.

24. Stahl M, Ries J, Vermeulen J, Yang H, Sham HP, Crowley SM, Badayeva Y, Turvey SE, Gaynor EC, Li X, et al.: A Novel Mouse Model of Campylobacter jejuni Gastroenteritis Reveals Key Proinflammatory and Tissue Protective Roles for Toll-like Receptor Signaling during Infection. PLoS Pathog 2014, 10:e1004264. [PubMed: 25033044]

25. Arena ET, Campbell-Valois F-X, Tinevez J-Y, Nigro G, Sachse M, Moya-Nilges M, Nothelfer K, Marteyn B, Shorte SL, Sansonetti PJ: Bioimage analysis of Shigella infection reveals targeting of colonic crypts. Proc Natl Acad Sci 2015, 112:E3282-E3290. [PubMed: 26056271]

26. Pédron T, Nigro G, Sansonetti PJ: From homeostasis to pathology: decrypting microbe-host symbiotic signals in the intestinal crypt. Philos Trans R Soc B Biol Sci 2016, 371:20150500.

27. Croxen MA, Sisson G, Melano R, Hoffman PS: The Helicobacter pylori Chemotaxis Receptor TlpB (HP0103) Is Required for $\mathrm{pH}$ Taxis and for Colonization of the Gastric Mucosa. J Bacteriol 2006, 188:2656-2665. [PubMed: 16547053] 
28. Sweeney EG, Henderson JN, Goers J, Wreden C, Hicks KG, Foster JK, Parthasarathy R, Remington SJ, Guillemin K: Structure and Proposed Mechanism for the pH-Sensing Helicobacter pylori Chemoreceptor TlpB. 2012, doi:10.1016/j.str.2012.04.021.

29. Huang JY, Goers Sweeney E, Guillemin K, Amieva MR: Multiple Acid Sensors Control Helicobacter pylori Colonization of the Stomach. PLoS Pathog 2017, 13:e1006118. [PubMed: 28103315] This work observed that $H$. pylori uses TlpA and TlpD to sense acid. $\Delta t l p A D$ mutants have defects colonizing the gastric glands and this defect is restored upon treatment with proton pump inhibitor to inhibit acid secretion.

30. McColl K, El-Omar E, Gillen D: Interactions between H. pylori infection, gastric acid secretion and anti-secretory therapy. Br Med Bull 1998, 54:121-138. [PubMed: 9604437]

31. Donaldson GP, Lee SM, Mazmanian SK: Gut biogeography of the bacterial microbiota. Nat Rev Microbiol 2015, 14:20-32. [PubMed: 26499895]

32. Tropini C, Earle KA, Huang KC, Sonnenburg JL: The Gut Microbiome: Connecting Spatial Organization to Function. Cell Host Microbe 2017, 21:433-442. [PubMed: 28407481]

33. Ermund A, Schutte A, Johansson MEV, Gustafsson JK, Hansson GC: Studies of mucus in mouse stomach, small intestine, and colon. I. Gastrointestinal mucus layers have different properties depending on location as well as over the Peyer's patches. AJP Gastrointest Liver Physiol 2013, 305:G341-G347.

34. Taniguchi Y, Kimura K, Satoh K, Yoshida Y, Kihira K, Takimoto T, Saifuku K, Ido K, Ookawara S, Mato M: Helicobacter pylori detected deep in gastric glands: An ultrastructural quantitative study. J Clin Gastroenterol 1995, 21:S169-S173. [PubMed: 8775013]

35. Ogura Y, Lala S, Xin W, Smith E, Dowds TA, Chen FF, Zimmermann E, Tretiakova M, Cho JH, Hart J, et al.: Expression of NOD2 in Paneth cells: a possible link to Crohn's ileitis. Gut 2003, 52:1591-7. [PubMed: 14570728]

36. Petnicki-Ocwieja T, Hrncir T, Liu Y-J, Biswas A, Hudcovic T, Tlaskalova-Hogenova H, Kobayashi $\mathrm{K}$ : Nod2 is required for the regulation of commensal microbiota in the intestine. PNAS 2009, 106:15813-15818. [PubMed: 19805227]

37. Foltz CJ, Fox JG, Cahill R, Murphy JC, Yan L, Shames B, Schauer DB: Spontaneous inflammatory bowel disease in multiple mutant mouse lines: association with colonization by Helicobacter hepaticus. Helicobacter 1998, 3:69-78. [PubMed: 9631303]

38. Handa O, Naito Y, Yoshikawa T: Helicobacter pylori: A ROS-inducing bacterial species in the stomach. Inflamm Res 2010, 59:997-1003. [PubMed: 20820854]

39. Collins KD, Andermann TM, Draper J, Sanders L, Williams SM, Araghi C, Ottemann KM: The Helicobacter pylori CZB cytoplasmic chemoreceptor TlpD forms an autonomous polar chemotaxis signaling complex that mediates a tactic response to oxidative stress. J Bacteriol 2016, 198:15631575. [PubMed: 27002127]

40. Collins KD, Hu S, Grasberger H, Kao JY, Ottemann KM: Chemotaxis Allows Bacteria To Overcome Host-Generated Reactive Oxygen Species That Constrain Gland Colonization. 2018, doi:10.1128/IAI.00878-17.This study found that $\Delta t 1 p D$ mutants that cannot sense reactive oxygen species had defects spreading into new gastric glands. This defect was rescued when $\Delta t l p D$ mutants were used to infect mice that were deficient in the production of reactive oxygen species.

41. Mazmanian SK, Cui HL, Tzianabos AO, Kasper DL: An immunomodulatory molecule of symbiotic bacteria directs maturation of the host immune system. Cell 2005, 122:107-118. [PubMed: 16009137]

42. Round JL, Lee SM, Li J, Tran G, Jabri B, Chatila TA, Mazmanian SK: The Toll-Like Receptor 2 Pathway Establishes Colonization by a Commensal of the Human Microbiota. 2010, doi:10.1126/ science.1198719.B. fragilis mutants that lack polysaccharide A (PSA) were seen to cause an altered immune response compared to WT. This observation was associated with PSA mutants being unable colonize the colonic crypts.

43. Stahl M, Vallance BA: Insights into Campylobacter jejuni colonization of the mammalian intestinal tract using a novel mouse model of infection. Gut Microbes 2015, 6:143-148. [PubMed: 25831043]

44. Stahl M, Frirdich E, Vermeulen J, Badayeva Y, Li X, Vallance BA, Gaynor EC: The helical shape of Campylobacter jejuni promotes in vivo pathogenesis by aiding transit through intestinal mucus 
and colonization of crypts. Infect Immun 2016, 84:3399-3407. [PubMed: 27647867] Curved-rod mutants of $C$. jejuni that retain partial helical shape were seen to lose motility in soft agar but not in liquid media. These curved-rod mutants were also unable to colonize the cecal crypts compared to WT $C$. jejuni.

45. Neumann CS, Richmond MH: Adhesion of commensal bacteria to the large intestine wall in humans. Infect Immun 1979, 23:128-132. [PubMed: 370006]

46. Davis CP, Mulcahy D, Takeuchi A, Savage DC: Location and description of spiral-shaped microorganisms in the normal rat cecum. Infect Immun 1972, 6:184-192. [PubMed: 4120246]

47. Phillips M, Lee A, Leach WD: The mucosa-associated microflora of the rate intestine: a study of normal distribution and magnesium sulphate induced diarrhoea. AjEBAK 1978, 56:649-662. [PubMed: 752307]

48. Keilberg D, Ottemann KM: How Helicobacter pylori senses, targets and interacts with the gastric epithelium. Environ Microbiol 2016, 18:791-806. [PubMed: 26768806]

49. Johnson KS, Ottemann KM: Colonization, localization, and inflammation: the roles of H. pylori chemotaxis in vivo. Curr Opin Microbiol 2018, 41:51-57. [PubMed: 29202336]

50. Howitt MR, Lee JY, Lertsethtakarn P, Vogelmann R, Joubert LM, Ottemann KM, Amieva MR: Chepep controls helicobacter pylori infection of the gastric glands and chemotaxis in the epsilonproteobacteria. MBio 2011, 2.This study the first to demonstrate that chemotaxis was important for gastric gland colonization. Howitt et al. determined that chemotaxis protein ChePep is required for chemotaxis and ChePep mutants have defects colonizing the gastric glands.

51. Cerda O, Rivas A, Toledo H: Helicobacter pylori strain ATCC700392 encodes a methyl-accepting chemotaxis receptor protein (MCP) for arginine and sodium bicarbonate. FEMS Microbiol Lett 2003, 224:175-181. [PubMed: 12892880]

52. Butler SM, Camilli A: Going against the grain: Chemotaxis and infection in Vibrio cholerae. Nat Rev Microbiol 2005, 3:611-620. [PubMed: 16012515] 


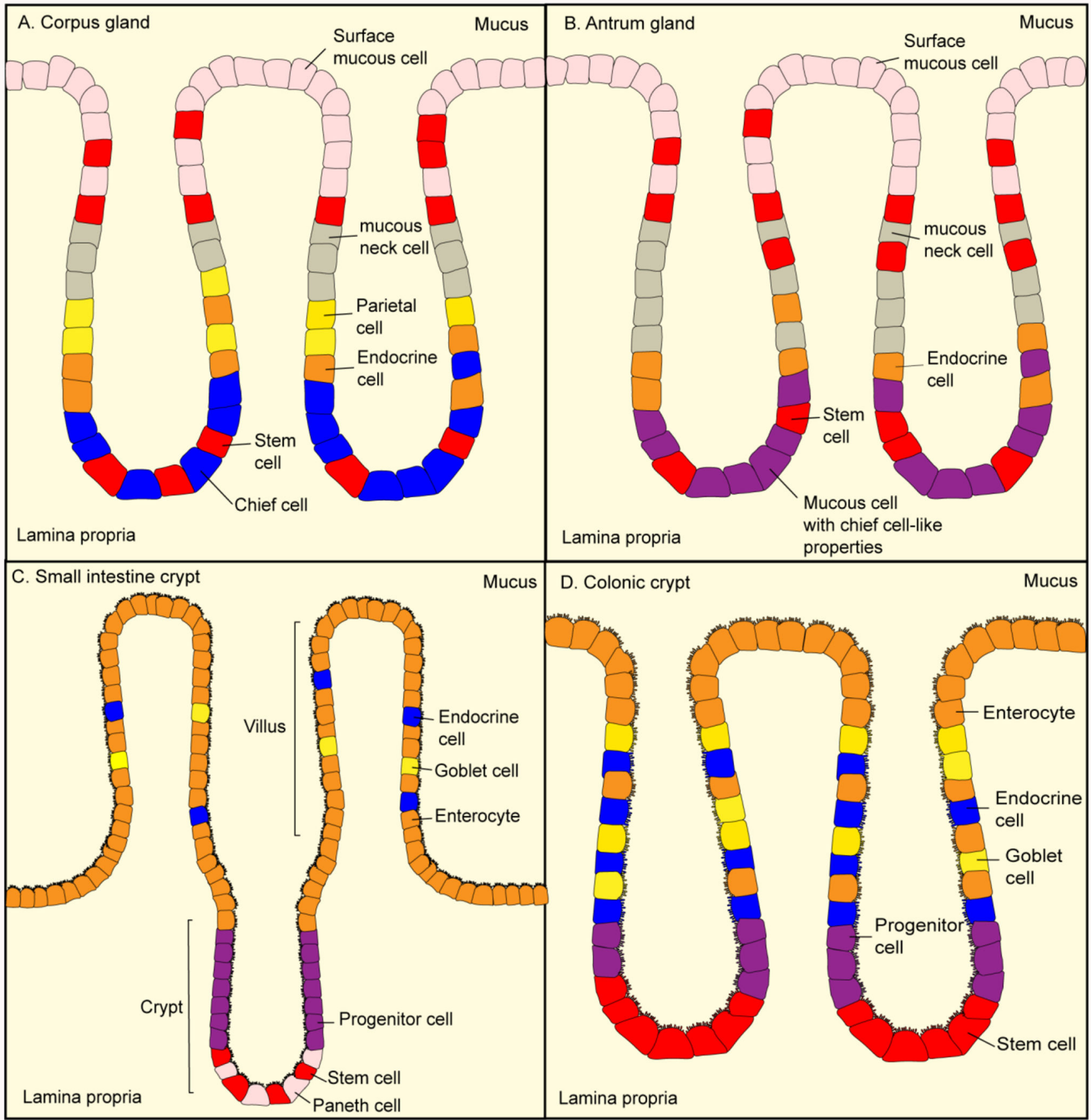

Figure 1.

Gland and crypt structures and cellular composition throughout the GI tract. A. Gland of the gastric corpus, consisting of surface mucous cells (pink), mucous neck cells (gray), parietal cells (yellow), endocrine cells (orange), chief cells (blue), and stem cells (red). The stem cells can differentiate into the different subtypes of cells that belong in the corpus gland. Parietal cells produce acid. Surface mucous and mucous neck cells produce mucus that protects the epithelial cells from the acid. Chief cells produce the digestive zymogen, pepsinogen. Endocrine cells produce different hormones to signal processes that occur in the 
stomach. B. Gland of the gastric antrum. The antrum gland has fewer cell types compared to the corpus gland. There are stem cells (red) that differentiate into the different cell types in the antrum gland. There are surface mucous cells (pink) and mucous neck cells (gray) that produce mucin. Endocrine cells (orange) produce hormones that regulate different processes in the stomach. C. Crypt of the small intestine. The small intestine crypts have finger-like projections called villi to increase the surface area of the small intestine. At the base of a villus, the epithelial cell layer invaginates to form a crypt. Each individual cell in the crypt has more finger-like projections called microvilli to further increase the surface area of the small intestine. Within the crypt, there are stem cells (red) at the base that differentiate into the different cell types of the crypt. There are also Paneth cells (pink) that secrete antimicrobial substances to regulate microbial colonization in the small intestine. There are also progenitor cells that differentiate into a subset of the cells that are in the crypt. In the villus are enterocytes (orange), goblet cells (yellow), and endocrine cells.(blue). The enterocytes function to absorb digested nutrients. Goblet cells produce mucus to protect the epithelial cell layer. The endocrine cells function to produce hormones to regulate processes that occur in the small intestine. D. Crypt of the colon/large intestine. In the colon, there are no villi, but the cells have microvilli to increase surface area for absorption. Similar to the small intestine crypt, there are stem cells (red) and progenitor cells (purple) near the base of the crypt that give rise to the different cell types in the colonic crypt. There are also goblet cells (yellow), enterocytes (orange), and endocrine cells (blue) that function in the same manner as they do in the small intestine crypt. 